16 | 2020

Les marges créatrices : intellectuel.le.s afrodescendant.e.s et indigènes auX Amériques, XIX-XXe siècle

\title{
De la marge à l'avant-garde : Conversation From Life d'Alice Childress ou la promotion de la lutte des domestiques noires
}

From the Margin to the Vanguard: Conversation From Life by Alice Childress Promoting the Black Domestic Workers' Struggle

De los márgenes a la vanguardia: Conversation From Life de Alice Childress o la promoción de la lucha de las trabajadoras domésticas negras

\section{Grégory Bekhtari}

\section{OpenEdition}

1 Journals

Édition électronique

URL : http://journals.openedition.org/ideas/8782

DOI : $10.4000 /$ ideas.8782

ISSN : 1950-5701

Éditeur

Institut des Amériques

Référence électronique

Grégory Bekhtari, « De la marge à l'avant-garde : Conversation From Life d'Alice Childress ou la promotion de la lutte des domestiques noires ", IdeAs [En ligne], 16 | 2020, mis en ligne le 01 octobre 2020, consulté le 18 octobre 2020. URL : http://journals.openedition.org/ideas/8782 ; DOI : https:// doi.org/10.4000/ideas.8782

Ce document a été généré automatiquement le 18 octobre 2020.

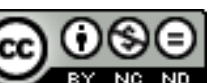

IdeAs - Idées d'Amériques est mis à disposition selon les termes de la licence Creative Commons Attribution - Pas d'Utilisation Commerciale - Pas de Modification 4.0 International. 


\section{De la marge à l'avant-garde : Conversation From Life d'Alice Childress ou la promotion de la lutte des domestiques noires}

From the Margin to the Vanguard. Conversation From Life by Alice Childress Promoting the Black Domestic Workers' Struggle

De los márgenes a la vanguardia: Conversation From Life de Alice Childress o la promoción de la lucha de las trabajadoras domésticas negras

\section{Grégory Bekhtari}

\section{Introduction}

1 Dans What Moves at the Margins, l'écrivaine Toni Morrison relate les difficultés qu'elle a connues à l'âge de douze ans, alors qu'elle faisait quelques heures de ménage pour une femme blanche, pendant la Seconde Guerre Mondiale. Elle était au départ peu efficace, puis apprit à bien nettoyer la maison ; «si bien qu'Elle me donnait plus de choses à faire, beaucoup plus. [...] Si je refusais, Elle me renverrait. [...] J'avais encore du mal à trouver le courage de discuter ou de m'opposer aux demandes croissantes qui m'étaient faites $»^{1}$. Se sentant piégée dans un rapport de pouvoir favorable à la femme blanche qui l'emploie, elle se tourne un jour vers son père pour sortir de son isolement. C'est au détour d'une conversation en prenant le café avec lui dans la cuisine qu'elle obtient de sa part les conseils et le soutien dont elle a besoin et qu'elle ne peut trouver nulle part ailleurs. À l'image de ce témoignage, négocier les conditions de travail avec l'employeuse blanche, défendre une dignité menacée par des abus de pouvoir dans le cadre d'une relation interpersonnelle hiérarchisée et sans l'appui d'une structure collective pour faire valoir ses droits relève du vécu quotidien de très nombreuses 
femmes noires ayant exercé le métier de domestiques aux États-Unis depuis la proclamation de l'émancipation des esclaves en 1863.

Bien avant que Morrison ne nous dévoile cette conversation avec son père dans son récit autobiographique, Alice Childress rédigeait Conversation From Life, une série de textes qui prend pour objet la dimension centrale de l'histoire d'un nombre très important de femmes noires américaines dans le contexte particulier du début des années 1950. Comédienne et auteure africaine-américaine née en 1916 à Charleston en Caroline du Sud, Childress a écrit des pièces de théâtre et des romans. Elle est surtout connue aujourd'hui pour A Hero Ain't Nothin' but A Sandwich, devenu un classique de la littérature de jeunesse aux États-Unis et adapté au cinéma en 1978. La première partie de sa carrière est restée méconnue jusqu'à ce que la spécialiste de littérature africaineaméricaine Mary Helen Washington ne la mette en lumière au début des années $2000^{2}$. La chercheuse révèle notamment ses affinités avec le Parti Communiste des États-Unis (CPUSA) ainsi que son appartenance à un réseau d'artistes et d'intellectuel.le.s noir.e.s militant.e.s proches du parti et dont le célèbre comédien, chanteur et militant des droits civiques Paul Robeson est une des figures emblématiques au début des années 1950. Or, dès juin 1949, parce qu'il s'est rendu au Congrès pour la Paix à Paris et a déclaré publiquement son amitié avec l'URSS en pleine guerre froide, Robeson se retrouve mis au ban des grands médias. Du côté de la presse noire, The Crisis, le magazine de la NAACP, le boycotte puis l'attaque ${ }^{3}$. Il devient nécessaire pour lui d'avoir son propre organe de presse pour se défendre et continuer à diffuser son discours en faveur de la libération des Noirs, aux États-Unis comme en Afrique, et en soutien aux syndicalistes et aux militant.e.s communistes ciblé.e.s comme lui par la propagande maccarthyste qui fait rage. Par amitié pour Robeson mais surtout parce qu'elle partage ses valeurs et ses convictions, Childress décide, à partir de septembre 1951, de participer à son mensuel nommé Freedom en hommage au premier journal noir fondé en $1827^{4}$, et ce jusqu'à l'arrêt de la publication en août 1955 .

3 Conversation From Life, sa rubrique qui paraît à chaque numéro sur deux colonnes, le plus souvent en dernière page (8), en miroir de la rubrique de Robeson qui se trouve en première page, est un récit de la vie quotidienne de Mildred, domestique noire habitant à Harlem, sous forme de dialogue avec son amie et voisine Marge, domestique, elle aussi. Par sa profession, Mildred appartient à une marge sociale. Et comme Childress la dépeint en sympathisante des idées de Robeson, en membre de la famille « Freedom », elle peut aussi être classée à la marge politique dans une période où ces idées progressistes sont couramment qualifiées d'anti-américaines et où celles et ceux qui les prônent peuvent être harcelé.e ss, voire persécuté.e.s. C'est pourtant depuis cet espace de la marge que Mildred tente de populariser la culture politique à laquelle elle est affiliée, et elle y parvient assez bien, au moins dans les limites de la communauté noire. En effet, la rubrique passe du mensuel Freedom, qui a au maximum 25000 lecteurs ${ }^{5}$, à l'hebdomadaire Baltimore Afro-American, le second plus grand journal noir des ÉtatsUnis, qui tire à 236000 exemplaires en $1949^{6}$, et où elle paraît de 1956 à 1959 , sous le nouveau titre de Here's Mildred.

Dans From Mammies to Militants, l'historienne de la littérature Trudier Harris propose une lecture critique de Like One of the Family, un recueil des rubriques publié en 1956. Optant pour une perspective diachronique, Harris ancre l'ouvrage dans une tradition artistique de représentation des domestiques noires par des auteur.e.s noir.e.s comme Charles Waddell Chesnutt, Ann Petry, Richard Wright ou Kristin Hunter et explique en 
quoi Childress se détache de cette tradition qui continue d'ailleurs après la parution du recueil. Selon elle, le personnage de Mildred s'éloigne du stéréotype de la mammy qui incarne l'obéissance et la fidélité à la famille blanche qui l'emploie ${ }^{7}$. La domestique représentée par Childress ne passe pas non plus par des biais détournés pour contester l'autorité de ses patrons. Refusant la place périphérique qui lui est assignée dans la famille blanche qui l'emploie, où elle bénéficie au mieux d'un simulacre de lien de parenté 8 la figure de Mildred affirme son indépendance et sa centralité au sein de la communauté noire à laquelle elle revendique fièrement son appartenance.

Cet article envisage une approche complémentaire à la thèse d'Harris, en ce qu'il relève davantage de l'histoire sociale et politique. Il s'agira de démontrer que la série de courts textes publiés dans le journal Freedom de 1950 à 1955, en pleine guerre froide, peut être définie comme un projet politico-culturel de défense et de promotion des droits et des intérêts des femmes noires et en particulier des domestiques parmi elles. Dans cette perspective, nous examinerons dans un premier temps la démarche de Childress quand elle écrit Conversation From Life au prisme de son appartenance au Black Left Feminism (féminisme noir de gauche). Ce mouvement est constitué de militantes noires actives au sein des organisations liées au CPUSA à partir des années 1930, en lien avec la communauté noire9. L'expression pour le dénommer a été forgée par Mary Helen Washington afin d'inscrire les femmes engagées dans ce mouvement dans le cadre de la Vieille Gauche (old Left) tout en montrant dans quelle mesure leurs pratiques et leurs écrits étaient précurseurs du féminisme noir (Black Feminism) des années 1960-70. L'un des objectifs principaux de ce mouvement est de mettre la condition marginale des femmes noires, et en particulier les domestiques, au centre des préoccupations des mouvements ouvrier et de libération noire des années 1930 au milieu des années 1950 et de faire entendre leur voix. Dans un second temps, nous verrons que le projet éditorial du journal Freedom, propre à un collectif militant de la gauche noire marqué par le contexte de répression maccarthyste, permet d'accorder une place centrale à la parole des femmes noires, notamment par l'intermédiaire du personnage de Mildred. Enfin, nous nous pencherons sur la forme d'écriture originale choisie par l'auteure qui confère une autorité politique à la parole du personnage principal de la domestique. Ce faisant, elle rattache ses textes au champ de la littérature propagandiste, assume leur dimension subversive sur le plan esthétique aussi bien que pour les prises de position qu'elle revendique et les place à l'avant-garde, tant littéraire que politique.

\section{Le Black Left Feminism : un réseau politique né des effets de la Grande Dépression et source de création culturelle}

6 "Pourquoi nous avons besoin d'un syndicat ${ }^{10}$ ». C'est le titre de la rubrique Here's Mildred du 14 juin 1958. Mildred s'y adresse à Marge selon une répartition des rôles assez courante dans Conversation From Life, qui rappelle d'une certaine façon la maïeutique socratique. À chaque fois, dans le dialogue entre pairs, tout se passe comme si la fonction de l'héroïne était de faire émerger la conscience politique de sa voisine et amie, peu convaincue a priori par la légitimité d'un positionnement militant : « Marge, pourquoi est-ce que tu ris à chaque fois que je dis que les domestiques ont besoin d'un syndicat? Bien sûr qu'on en a besoin! ». Cette forme de dialogue horizontal permet de 
passer en revue les lieux communs et autres préjugés émis par Marge, qui tendent souvent à justifier l'ordre établi, pour mieux y répondre et espérer faire évoluer son point de vue de départ : "Oui, ma vieille, j'ai entendu parler des rares cas où certaines personnes ont laissé de l'argent à leurs domestiques sur leur testament [...] mais si nous avions un syndicat [...], nous n'aurions pas à dépendre de la générosité de qui que ce soit. » À l'opposé, le second type de conversation présente dans ces textes - le dialogue vertical qui oppose Mildred à ses patronnes blanches - n'est qu'un moyen de contester la supériorité et de limiter le pouvoir des personnes qui l'emploient, comme on le constate dans le numéro de janvier 1952 où la domestique répond par une fin de nonrecevoir à la requête écrite de la maîtresse de maison d'effectuer des tâches supplémentaires : «à l'avenir je vous prie de ne pas me laisser de notes contenant des demandes qui sortent du cadre de notre contrat...». Qu'elle traite de l'absence de couverture maladie et de retraite, de la protection que peuvent fournir les termes bien définis d'un contrat ou encore d'autres sujets liés à la condition des domestiques au travail, Childress s'y prête en ayant conscience qu'au-delà du contexte immédiat de la guerre froide, elle inscrit son récit dans une tradition de lutte de femmes noires au sein du mouvement ouvrier qui apparaît au début des années 1930, en réaction aux bouleversements sociaux de la Grande Dépression et dont le personnage central de Mildred est un point d'aboutissement.

7 Le réseau militant dont Childress fait partie émerge dans le sillage du National Negro Congress (NNC), une organisation qui luttait contre la discrimination raciale et en faveur des droits civiques, créée en février 1936 avec le soutien du CPUSA ${ }^{11}$. Son congrès de fondation met en avant un combat antiraciste qui privilégie la mobilisation de masse, le travail dans et avec les syndicats et le mouvement ouvrier en général, et qui défend un programme économique et social à la gauche des réformes du New Deal ${ }^{12}$.

De nombreuses militantes qui souhaitent mettre en lumière la question de la condition socio-économique des femmes noires aux États-Unis, reléguée jusque-là à la marge du débat politique, y trouvent alors une tribune. Elles sont en effet mues par une forme d'urgence face à une situation dramatique. Comme le rappelle Jean Collier Brown dans un rapport qu'elle produit pour le Bureau des affaires féminines du Ministère du travail, dès 1930, le chômage des femmes noires croît encore plus fortement que celui des autres catégories de la population active et elles sont alors 1150000 à exercer le métier de domestique, soit cinquante pour cent de plus qu'en $1920^{13}$. Pire, en 1935, les domestiques sont exclues des droits à la couverture maladie et à la pension actés par le Social Security Act impulsé par l'administration du New Deal ${ }^{14}$. Pendant le congrès, les débats ont lieu dans le cadre d'une sous-commission sur les droits des femmes. Le rapport qui en est tiré analyse la spécificité de leur oppression et insiste sur le sort des domestiques en leur sein :

Les femmes noires d'Amérique subissent une triple exploitation en tant que femmes, en tant que travailleuses et en tant que noires, et se retrouvent forcées à cause des discriminations à exercer les emplois les plus ingrats dans les pires conditions et sans qu'aucune protection n'ait été instituée. [...] Il est proposé : qu'un mouvement national soit lancé sous la direction du NNC pour que les domestiques, qui représentent $85 \%$ des femmes noires qui travaillent, rejoignent une organisation qui serve d'outil pour soutenir et promouvoir des lois [...] afin de garantir [...] l'égalité complète des femmes noires avec toutes les autres travailleuses ${ }^{15}$. 
Cette plateforme est pour ainsi dire l'acte de naissance du féminisme noir de gauche. La responsable communiste Louise Thompson ${ }^{16}$, elle-même présente lors du congrès de fondation du NNC et active au sein de la sous-commission sur les droits des femmes, fait un compte rendu des travaux et reprend la notion de triple exploitation dans un article de Woman Today, le journal du CPUSA destiné au lectorat féminin. Elle l'illustre en faisant référence au «marché aux esclaves du Bronx » (Bronx Slave Market), un célèbre lieu d'embauche des domestiques à New York où des femmes noires sans revenus attendaient qu'on les emploie à la journée :

Le marché aux esclaves du Bronx est un exemple sinistre de l'exploitation féroce de la fraction la plus exploitée de la population américaine : les femmes noires. [...] Partout dans le pays, les femmes noires subissent cette triple exploitation : en tant que travailleuses, en tant que femmes, et en tant que noires. Environ 85 pour cent de l'ensemble des femmes noires qui travaillent sont des domestiques, deux tiers des deux millions de domestiques que comptent les États-Unis ${ }^{17}$.

Dans cet article, Thompson met en scène les domestiques issues du marché aux esclaves du Bronx face à leurs potentielles patronnes blanches : «Prenez notre travail. Donneznous ce que vous pouvez. Nous devons nourrir nos enfants et payer notre loyer élevé à Harlem. 10, 15 cents de l'heure ? Vous ne payerez pas plus ? $»^{18}$.

11 Les effets de la Grande Dépression se faisaient sentir à tel point que les femmes noires étaient prêtes à accepter de travailler à des taux horaires extrêmement bas.

12 Au moment où la militante publie son texte, le marché aux esclaves du Bronx a déjà été mentionné l'année précédente dans un article paru dans The Crisis, co-signé par la militante du mouvement pour les droits civiques Ella Baker et la journaliste Marvel Cooke, elle aussi membre du CPUSA ${ }^{19}$. Pour faire entendre la cause des domestiques noires, Cooke avait produit une série de reportages ${ }^{20}$, après avoir travaillé un temps comme domestique. En tant que militante, elle collabore avec des syndicalistes qui les défendent et soutient leur organisation, le Domestic Workers Union (DWU ${ }^{21}$.

13 La reconnaissance et l'amélioration des conditions de travail et de vie des domestiques noires sont également un objectif essentiel d'Esther Cooper Jackson, responsable du Southern Negro Youth Congress (SNYC), l'organisation de jeunesse du NNC établie dans le Sud des États-Unis entre 1942 et 1946. En 1940, alors étudiante en master à l'université de Fisk, Jackson rédige la première étude sociologique consacrée aux domestiques noires et à leur rapport au syndicalisme : The Negro Woman Domestic Worker in Relation to Trade Unionism. Dans ce mémoire, elle souligne l'engagement concret du NNC en faveur de cette cause. Ce dernier a défendu l'accès à la couverture sociale des domestiques devant la Chambre des Représentants en mars 1939 et finance la section du DWU de Washington D.C., dont le siège est une salle qui se trouve dans ses propres locaux $^{22}$. Dans sa conclusion sur le travail domestique, Cooper insiste sur le fait que les femmes noires sont moins bien payées et travaillent dans des conditions bien pires que les employées blanches et elle établit un lien entre leur situation et leur rôle à l'avantgarde de l'action syndicale ${ }^{23}$. Elle a en effet constaté que seules des femmes noires animent les sections qu'elle a observées pour son étude.

14 Elle aborde également dans son travail de recherche ${ }^{24}$ la question du stigmate associé au métier de domestique, autrement dit l'infériorité sociale dont souffrent les personnes qui exercent cette profession. Cette question est de la première importance pour comprendre les ressorts du racisme tels qu'il s'impose à toutes les femmes noires aux États-Unis, au-delà des seules domestiques. 

stigmate dénoncé par Cooper trouve sa place parmi d'autres stéréotypes dévalorisants (dont celui de la Jezebel, femme à la sexualité débordante et agressive) ; la revendication d'une représentation à égalité avec les hommes et les femmes blanches dans les productions culturelles et en particulier dans les médias de masse (cinéma, presse, théâtre, radio...) est aussi un élément essentiel du combat des militantes issues du féminisme noir de gauche. Depuis sa création, le NNC a d'ailleurs un Département des Affaires Culturelles (Cultural Division) dont le but est de promouvoir les artistes noir.e.s et leurs œuvres, d'ouvrir de nouveaux horizons aux jeunes générations en termes de carrières et plus largement de soutenir la diffusion de représentations des Africain.e.s Américain.e.s affranchies des préjugés malveillants et/ou condescendants des blancs, aussi bien dans la littérature qu'au théâtre et au cinéma. La militante Thelma Dale insiste ainsi dès 1940 sur l'absence de perspective de reconnaissance sociale de la masse des jeunes femmes noires, notamment dans le domaine des arts et de la culture, au sein d'une société qui ne conçoit pour elles que la fonction de domestique et des conditions de travail déshumanisantes ${ }^{25}$. Devenue secrétaire administrative du NNC à partir de 1943, elle dénonce en 1947 la relégation des femmes noires aux emplois de domestiques, comme si celles-ci n'avaient pas leur place dans d'autres secteurs professionnels ${ }^{26}$. dirigeante du CPUSA Claudia Jones se livre à une analyse de la situation des femmes noires en contexte, non sans revenir sur la spécificité de leur histoire aux États-Unis. Publié dans Political Affairs, la revue théorique du parti, son texte a pour objectif de dénoncer leur marginalisation sociale et politique. Jones veut convaincre la direction du CPUSA que ces femmes peuvent jouer un rôle d'avant-garde pour transformer la société, précisément parce qu'elles sont victimes d'une triple exploitation et qu'elles sont nombreuses à avoir appris à résister et à s'organiser pour lutter contre ses effets. Elle s'attarde elle aussi sur l'importance de la lutte contre les clichés racistes véhiculés par les médias de masse autour de la figure de la domestique noire :

Le rapport essentiellement économique des femmes noires aux femmes blanches, qui perpétue les relations du type "Madame-et-sa-bonne », nourrit les attitudes racistes. [...] Au cinéma, à la radio, et dans la presse, la femme noire n'est pas représentée dans son rôle véritable, à savoir tout à la fois mère, personne qui subvient aux besoins de la famille, et personne qui la protège, mais dans le rôle traditionnel de la «mammy » qui place la satisfaction des besoins des enfants et des familles des autres au-dessus des siens. Ce stéréotype traditionnel de la mère esclave noire, qui jusqu'à aujourd'hui apparaît dans les publicités, doit être combattu et rejeté en tant qu'instrument aux mains des impérialistes pour perpétuer l'idéologie raciste des blancs selon laquelle les femmes noires sont " arriérées 》, « inférieures », et sont les « esclaves par nature » des autres ${ }^{27}$.

17 Elle ajoute que " les travailleurs progressistes du monde de la culture » doivent « relever le défi de la lutte contre le racisme » posé par cette injustice et « célébrer par leurs écrits et leurs chants la femme noire, son courage absolu et sa dignité ${ }^{28}$. La lutte contre la triple exploitation, qui se mène bien sûr sur les lieux de travail pour faire changer concrètement les conditions de vie des femmes noires, ne peut pas se passer non plus d'un combat idéologique mené par des créateurs et créatrices qui donnent à entendre des récits alternatifs aux mythes dominants qui légitiment au quotidien le racisme, le sexisme et l'exploitation. Alice Childress, qui partage cette préoccupation, prend l'enjeu au sérieux. La même année, elle écrit sa première pièce, Florence, au sujet d'une femme noire et pauvre qui décide de quitter la Caroline du Sud pour s'installer à 
New York et devenir actrice, et qui refuse d'être assignée aux rôles de domestique ${ }^{29}$ qu'on lui propose. La dramaturge rejoint le courant féministe noir de gauche au moment où elle se lance officiellement dans l'écriture et la direction d'acteurs, après avoir été comédienne pendant plusieurs années. Elle devient aussi active au sein du Committee for the Negro in the Arts (CNA), nouvelle dénomination du Département des Affaires Culturelles du NNC après sa dissolution. Elle met en scène la première production de la troupe théâtrale du CNA, la pièce Just Simple, qu'elle adapte de l'œuvre de Langston Hugues ${ }^{30}$. C'est également au sein du CNA qu'elle rencontre l'acteur et chanteur Paul Robeson, pour le journal duquel elle commence à écrire la rubrique Conversation From Life à partir de septembre 1951.

\section{Freedom : un porte-voix de la parole des femmes noires}

18 À travers la figure connue de Robeson, l'objectif du journal Freedom lancé dès novembre 1950 est de consolider des alliances entre la communauté noire et le mouvement ouvrier progressiste et antiraciste pour résister à la vague maccarthyste. L'arrêt de la publication en janvier 1955 marque l'échec de cet objectif face à l'hégémonie culturelle anticommuniste qui s'est irrésistiblement installée au sein de la société étatsunienne et pousse de nombreux kiosques à refuser de vendre le journal ${ }^{31}$. Il était par ailleurs de plus en plus difficile de financer ce journal, dont le lectorat progressiste était plus restreint que celui des grands titres de la presse noire. Malgré l'apport des économies de Robeson, les abonnements, les souscriptions et les nombreux concerts que le chanteur a organisés pour récolter des fonds, comme celui du Peace Arch Park à la frontière canadienne qui a rassemblé 40000 personnes et dont le numéro de juin 1952 de Freedom s'est fait l'écho, les fonds levés n'ont pas suffi à couvrir les dépenses.

Cependant ce projet journalistique a malgré tout contribué à faire sortir de la marge la parole des femmes noires et celle des domestiques noires par la même occasion, tout au moins au sein de la communauté noire et du mouvement ouvrier où elles peinaient à se faire entendre. Deux raisons expliquent cette réussite. La première, c'est la présence au sein du comité de rédaction et des auteur.e.s régulièr.e.s de nombreuses femmes, affiliées au courant du féminisme noir de gauche : la journaliste Yvonne Gregory, la syndicaliste Vicki Garvin, l'anthropologue Eslanda Robeson, l'écrivaine Lorraine Hansberry, l'auteure et compositrice Shirley Graham Du Bois et bien sûr Alice Childress. Leurs articles et reportages ainsi que leur participation aux choix éditoriaux garantissent que le point de vue et la condition des femmes noires soient des thèmes incontournables du mensuel. Elles soutiennent par exemple Sojourners For Truth and Justice (STJ), une organisation de femmes noires qui lutte à la fois contre le racisme, contre le maccarthysme et pour la dignité des femmes noires, en promouvant ses initiatives dans les pages de Freedom ${ }^{32}$. À l'automne 1951, au moment où elle débute sa collaboration au journal, Childress signe d'ailleurs l'appel à créer le STJ aux côtés de Robeson, Graham Du Bois ou encore Louise Thompson ${ }^{33}$. La seconde raison, c'est la volonté et la nécessité de développer une base de soutien et un lectorat les plus larges possibles, pour mieux influencer les classes populaires qui forment la très grande majorité de la population noire ${ }^{34}$. Les domestiques noires étaient donc de fait une cible privilégiée. 
20 Avant même la première parution de Conversation From Life, on trouve en page deux du numéro de mars 1951 un dessin humoristique mettant en scène une employée de maison noire face à ses employeurs. Sa patronne s'adresse à elle en ces termes : " Ophélia, Mr. Roberts et moi, nous faisons notre part pour dévoiler les mensonges des Rouges à propos de notre démocratie. Donc vous n'avez plus besoin d'entrer par la porte de derrière. Nous allons en faire construire une pour vous sur le côté ". Le regard méfiant d'Ophélia à l'écoute de ces propos est symbolique du message critique de Freedom adressé à la communauté noire : il ne faut pas faire confiance aux discours hypocrites et mensongers des blancs qui les maintiennent en situation d'infériorité, surtout quand ils prétendent agir pour leur bien. Ce dessin révèle l'objectif du comité de rédaction, être audible auprès des domestiques ; passer par l'intermédiaire du récit régulier de la vie d'un personnage de domestique, certes fictionnel mais dont la dimension réaliste a des accents documentaires, a pu sembler le meilleur moyen d'y parvenir par la suite.

21 L'analyse de Mary Helen Washington peut nous aider à mieux définir la place stratégique que cette rubrique occupait au sein du mensuel. Washington insiste sur la « relation dialogique » que la rubrique entretient « avec les autres histoires et écrivains du journal ${ }^{35}$ » et la considère comme un " texte collaboratif » en cohérence avec " l'objectif du journal de développer l'information politique au sein de la communauté noire ${ }^{36} »$. En effet, les discours de Mildred faisaient écho, tout en les résumant au moyen d'un langage accessible qui tranchait avec le jargon militant, aux enjeux principaux traités dans les articles de fond de Freedom : la guerre de Corée (février 1953) et plus largement la lutte en faveur de la paix en temps de guerre froide (janvier 1954), le syndicalisme (septembre 1953), l'histoire et la culture africaines-américaines (février 1952), l'anticolonialisme et la solidarité internationale avec les luttes de libération nationale, notamment en Afrique (juin 1953), le combat pour les droits civiques (décembre 1951, avril 1953) et la dénonciation du maccarthysme et de la propagande anticommuniste (mars 1952, janvier 1954).

La parution de la première rubrique en septembre 1951 est représentative de cet objectif. Cette dernière se trouve à la page qui suit l'article « Poet Demands Equality For Negro Womanhood" qui présente le poème «A Black Woman Speaks of White Womanhood, of White Supremacy, of Peace » écrit par l'actrice Beulah Richardson. Ce poème vient d'être primé par un jury, dont Alice Childress était membre, lors d'un congrès pour la paix tenu par l'American Peace Crusade, une organisation proche du CPUSA qui prône la fin de la guerre froide ${ }^{37}$. Il dénonce la féminité blanche comme norme sociale responsable de la position subalterne des femmes noires. Le sujet lyrique, une femme noire représentant l'ensemble de ses semblables, tente de convaincre les femmes blanches de se détacher de l'idéologie de la suprématie blanche et de devenir des alliées dans le combat pour l'égalité ${ }^{38}$.

La situation à laquelle le personnage de Mildred fait face, dans la rubrique qui suit, est une transposition concrète des rapports hiérarchiques entre femmes blanches et femmes noires mis à nu dans le texte de Richardson dans le cadre spécifique au travail de domestique. La nouvelle patronne de Mildred, Mrs. James, parle à la troisième personne quand elle lui donne des instructions, pour mieux se dissocier de sa domestique noire et lui signifier sa supériorité. Pour rétablir une forme d'égalité rhétorique, Mildred fait de même en lui faisant savoir qu'elle refuse de travailler le samedi. Mécontente de voir son employée sortir si aisément de la place inférieure 
qu'elle veut lui assigner, Mrs. James lui fait plus tard cette remarque : «Mildred, une dépression pourrait faire du bien à ce pays, alors certaines personnes travailleraient huit jours par semaine (sic) et seraient bien contentes de la chance qu'elles ont ", ce à quoi Mildred rétorque : «C'est vrai, mais d'un autre côté des gens se retrouveraient à faire le ménage eux-mêmes ». En utilisant le langage à son avantage, et en ayant le dernier mot face à sa patronne, Mildred fait résonner le message du poème de Richardson: prendre la parole face aux femmes blanches et obtenir d'elles qu'elles écoutent et qu'elles se taisent est une première étape sur le chemin de l'égalité.

Mais les courts récits de Childress ne sauraient être réduits à de la simple vulgarisation sur des sujets considérés comme essentiels par la rédaction. En complément à la figure de Paul Robeson, que le lectorat peut admirer pour ses talents artistiques et son engagement en faveur des droits civiques, mais à qui il est difficile de se comparer, l'auteure met en avant le personnage de Mildred qui incarne le lectorat auquel elle s'adresse afin qu'il soit reconnu comme la voix des classes populaires noires. Cette fonction emblématique est manifeste dans le numéro de février 1952. La rubrique intitulée "The Many Others in History " est consacrée à la Negro History Week, la semaine de célébration de l'histoire des Noirs aux États-Unis. Elle montre Mildred passant du statut d'auditrice à celui d'oratrice lors d'une conférence sur la vie et l'œuvre de personnages historiques comme Frederick Douglass, Nat Turner, Harriet Tubman et Sojourner Truth. À la fin des présentations, Mildred prend la parole pour raconter la vie et l'œuvre de sa grand-mère. Son exposé légitime la conception d'une histoire populaire africaine-américaine, dont les intellectuel.le.s ne sont pas les seul.e.s dépositaires. Celle-ci ne se limite pas aux grandes figures, car les chevilles ouvrières de la lutte pour la libération sont les gens ordinaires, dont une femme noire pauvre et anonyme comme la grand-mère de Mildred est l'archétype.

Enfin, le personnage de Mildred ne se contente pas de ressembler au public du journal pour créer un effet de familiarité et rendre sa lecture plus aisée et plus désirable, mais il est utilisé comme un véritable relais du lectorat. En mai 1952, Childress va jusqu'à mettre en scène Mildred comme une abonnée qui pointe les défauts du mensuel de son point de vue (pas de recettes de cuisine, de mots croisés ou de carnet mondain) mais finit par inciter tout le monde à participer à une souscription en sa faveur après avoir rencontré Paul Robeson. En juillet 1953, Mildred est même présentée comme une sorte d'ambassadrice du nouveau club des amis de Freedom, «Freedom Associate»: « J'ai particulièrement envie que tu nous rejoignes, Marge, parce que j'ai l'intention de faire quelques changements dans l'organisation et je vais avoir besoin que tu m'aides ». Cette initiative du journal pour être au plus près des modes de vie et de sociabilité de la communauté noire (fêtes, pique-niques, garden-parties, liens avec les églises...) est l'occasion de reconnaître, à travers Mildred, les femmes comme des organisatrices indispensables en son sein.

\section{« Des histoires de contestation » : de l'avant-garde littéraire à l'avant-garde politique}

Pour écrire Conversation From Life, Childress s'est très probablement inspirée des "Simple Stories» rédigées par Langston Hugues sous la forme d'une rubrique hebdomadaire dans le Chicago Defender à partir de $1943^{39}$. Les anecdotes vécues par le personnage principal Jesse B. Semple, un homme noir issu de la classe ouvrière qui 
passe son temps libre à raconter des histoires dans un bar de Harlem, sont selon l'historien de l'art Michael Denning "sa fiction "prolétarienne" la plus réussie ${ }^{40}$ ". L'éclairage de Denning peut nous permettre de mieux comprendre les textes de Childress et ses ambitions politico-esthétiques :

Le mouvement de la littérature prolétarienne s'inscrit dans une "guerre des classes » dans la littérature, [...] a émancipé une génération d'écrivains issus de la classe ouvrière et de diverses origines ethniques; $[. .$.$] leur a permis de représenter -$ de parler pour et de dépeindre - leurs familles, leurs quartiers, leurs aspirations. [...] Burke [...] suggérait que «les insistances et les exhortations du style "prolétarien" peuvent fournir un stimulus durable et essentiel à la formation de la "conscience" nationale $»^{41}$.

Nul doute que les textes de l'auteure publiés dans Freedom partageaient les caractéristiques et les objectifs de la littérature dite prolétarienne, enrichie du point de vue situé d'une femme noire. Le travail de mise en récit auquel Childress se livre à des fins de propagande politique n'est d'ailleurs pas propre à sa fiction. Il était déjà présent sous la plume d'autres membres du réseau féministe noir de gauche, comme Louise Thompson, qui animait le salon littéraire Vanguard pendant la Renaissance de Harlem $^{42}$, dans son article de 1936 « Towards a Brighter Dawn » qui fait s'exprimer les victimes du marché aux esclaves du Bronx. Dans le compte-rendu d'une conférence sur la condition des femmes organisée par le CPUSA à Pittsburgh en 1947 par exemple, Claudia Jones rapporte pour sa part la parole de 5 femmes. Sur 120 lignes d'entretiens, les 4 femmes blanches en occupent 80, et l'unique femme noire 40. Jones ne se contente pas de faire passer celle-ci de la marge, où elle est habituellement reléguée, au centre, elle la place également à l'avant-garde dans l'économie de son récit pour la représenter en position de meneuse :

Garnet est une domestique. Ses mots exprimaient la0 triple peine de la femme noire - le fardeau d'être une femme, d'être noire, d'être condamnée au service domestique sans alternative. [...] "Je suis très heureuse d'être ici. Je trouve formidable que d'autres femmes s'intéressent autant à nous, les femmes noires, et à nos problèmes spécifiques ». [...] Lorsque Garnet Johnson s'assit, il y eut un profond silence pendant une minute ou deux. Puis les autres femmes commencèrent à applaudir l'appel de Garnet à l'unité et à l'organisation pour mener les batailles communes à toutes les femmes et les batailles spécifiques aux femmes noires ${ }^{43}$.

Insérée dans un cadre d'émulation militante aussi bien qu'artistique, la production de Childress n'a pas de mal à trouver sa propre singularité. Sa rubrique prend la forme d'une scène où pour la première fois une domestique noire tient le rôle principal, sans que l'on se moque d'elle ni que sa raison d'être soit le bonheur de la famille blanche qui l'emploie, comme dans le Beulah Show, la série célèbre critiquée par Lorraine Hansberry dans les pages de Freedom ${ }^{44}$. Mildred se soucie du bien-être de ses proches et de ses voisin.e.s à Harlem ${ }^{45}$. De plus, on ne rit pas à ses dépens, mais avec elle, et elle n'hésite pas à utiliser l'humour pour prendre le dessus sur sa patronne : « Vous pensez me faire un compliment quand vous dites : "Nous ne la considérons pas comme une servante...", mais après avoir travaillé jusqu'à en être trempée de sueur à laver la salle de bain et la cuisine, faire les lits, laver la vaisselle [...], je n'ai vraiment pas le sentiment d'avoir été invitée chez vous pour le week-end. $»^{46}$. Dramaturge, Childress semble concevoir ses Conversations From Life comme autant de fragments d'une pièce qui n'a jamais été jouée à l'époque, faute de trouver le théâtre qui prendrait le risque de la présenter. Forme courte adaptée au support spécifique du journal, la rubrique en question est aussi une forme hybride, qui mélange les genres du récit romanesque et du théâtre. La théâtralité 
est un choix esthétique qui vient renforcer l'effet de réel auprès du lectorat peu lettré et accroître chez lui le sentiment que c'est bien à son milieu social et à son vécu que les récits font référence. Mais elle est également mise au service de l'objectif d'éducation politique des lectrices poursuivi par l'auteure. Selon Mary Helen Washington, "Childress souhaitait que Mildred [...] soit (comme elle) une porte-parole qui fournisse aux femmes noires une théorie du droit du travail, une permission de se rebeller et un vocabulaire pour s'exprimer contre l'injustice $»^{47}$. Les conversations entre voisines qu'entretiennent Mildred et Marge, toutes deux domestiques, dans leur cuisine, ne sont pas que des scènes typiques de la vie des classes populaires noires à Harlem. Elles sont aussi ce qui se rapproche le plus des discussions de vestiaire ou de déjeuner entre collègues quand on est comme elles isolé sur son lieu de travail. De plus, la double énonciation à l'œuvre quand Mildred raconte ses journées de travail lui permet de s'adresser en même temps à sa patronne blanche et à Marge, ou plus exactement à toutes les femmes noires susceptibles de lire la rubrique.

Mais la véritable innovation de Childress tient à son invention d'une structure de dialogue récurrente qui insiste sur la relation de pouvoir entre patronne blanche et domestique noire, que l'on pourrait appeler « rapport de forces conversationnel », un rapport de forces qui, à contre-courant de l'ordre établi, aboutit dans les récits à l'imposition d'un quasi monologue de Mildred qui réduit à chaque fois sa patronne blanche au silence en réagissant aux diverses manifestations de racisme ou de mépris de classe qu'elle subit. Malgré le rapport hiérarchique de race et de classe, le récit se termine toujours par l'établissement d'une situation d'égalité avec ses employeurs blancs. Même quand elle se met en colère contre ses employeurs au sujet de leur réaction à une agression raciste et qu'ils lui demandent de rentrer chez elle sous prétexte qu'elle ne serait pas en état de travailler, c'est elle qui décide quand elle part et pas avant de leur avoir donné une leçon sur leurs préjugés ${ }^{48}$. Ce schéma de résolution de conflit peut sembler relativement invraisemblable hors de la fiction, dans un contexte de ségrégation raciale et au sein d'une profession quasi exclue du droit du travail. Cependant la représentation des victoires de Mildred sur les personnes qui l'exploitent et l'oppriment suscitaient un véritable engouement.

La popularité de la rubrique est en effet indéniable auprès du lectorat de Freedom. En février 1952, le journal annonce qu'il a reçu de nombreuses lettres de félicitations pour «Merry Christmas, Marge!» la rubrique de décembre 1951 où Mildred imagine les États-Unis sans ségrégation. La remarque d'une jeune mère de famille de Boston, citée pour l'occasion, permet de mesurer l'influence du discours du personnage en termes de formation et de consolidation des convictions politiques: «Quand j'ai lu la chronique d'Alice Childress, je me suis rendu compte qu'elle avait mis le doigt sur ce à quoi je pensais et que je voulais vraiment pour mon enfant. C'est la paix dont parle Alice Childress à laquelle j'aspire pour mon enfant et sans compromis ! ». À la demande du lectorat, cette même rubrique est publiée à nouveau en décembre 1952. Preuve supplémentaire de la popularité de la rubrique, le contenu de Conversation From Life est ensuite repris sous un autre nom par le Baltimore Afro-American.

31 Certes l'exemplarité du personnage de Mildred a ses limites. Célibataire, sans enfants, elle travaille à la journée et change très souvent d'employeur. Ces conditions l'affranchissent de nombreuses contraintes et lui procurent une liberté et une marge de manœuvre dont sont dépourvues la majorité des domestiques noires. Mais si la force de son impact est réelle, c'est parce que Mildred fait appel à une subjectivité ou une 
conscience collective propre à toutes les domestiques noires, même celles, nombreuses, dont le comportement au travail est éloigné du sien. Face à la triple oppression qu'elles subissent au quotidien, Mildred nourrit chez les domestiques qui lisent ses histoires un sentiment de fierté noire, de dignité ouvrière et de pouvoir d'agir féminin. Cela ressort puissamment par exemple des propos qu'elle tient à Marge dans le numéro de septembre 1953 à propos des syndicats et de la reconnaissance des salarié.e.s : « Bon sang, les gens de la classe ouvrière sont les plus merveilleux qui soient sur la terre entière... ». Les autres femmes noires ainsi que les hommes noirs peuvent aussi se retrouver dans ses discours et ses actes. Le réalisme émancipateur de Childress n'est d'ailleurs pas dénué de racines très concrètes. Il trouve sa source dans le combat syndical d'une minorité active de domestiques, comme la syndicaliste Nena Evans ${ }^{49}$, ou sa propre tante Lorraine qui, selon l'auteure, " ont tenté de monter un syndicat de domestiques à la fin des années $1940 \aleph^{50}$. De plus, sa tante, toujours selon elle, face à ses employeurs, "exprimait ses sentiments quand elle ne pouvait pas les contenir ${ }^{51}$ ", à l'instar de Mildred pour qui elle a servi de modèle. Mais même au-delà des militantes syndicales, les actes de résistance n'étaient pas exceptionnels. En réponse aux stratégies de Mildred qu'elle proposait de suivre pour poser des limites face aux patronnes, l'auteure était "inondée de lettres » de domestiques lui rapportant " davantage d'histoires de contestation ", et des prouesses qui " étaient toujours plus grandes [que celles de Mildred], ou du moins elles voulaient qu'elles le soient ${ }^{52} »$. En dernier ressort, la popularité du positionnement avant-gardiste de Mildred face à ses employeurs, dans le sens où celle-ci peut représenter l'avant-garde de la classe ouvrière en lutte, malgré la pression pour la normalisation des comportements exercée par le maccarthysme y compris au sein de la population noire, s'explique par la capacité de Childress à transmettre la culture politique du mouvement ouvrier, et en particulier de sa sensibilité communiste. L'héroïne parvient en effet à transmettre cette culture comme si elle était familière à ses interlocutrices, au moyen de références propres à la culture africaine-américaine, notamment la religion dans son rapport au mouvement de libération noir. Ce travail de transposition est manifeste dans la rubrique de décembre 1953 où la domestique défend le droit à la dissidence et à la contestation en citant la bible comme argument d'autorité. Comme le dit son amie Nellie dans le numéro d'avril 1955 après l'avoir écoutée exposer son évangile progressiste : « Mildred, le dernier homme qui a enseigné ces choses s'est fait crucifier». Figure d'un syndicalisme par et pour les domestiques noires, alors que celles-ci en étaient quasi exclues jusque-là, Mildred a aussi l'ethos d'une guide qui éclaire la voie vers un monde nouveau, où les dernières seront les premières.

\section{Conclusion}

32 Le réseau de femmes noires qui donne corps au féminisme noir de gauche du milieu des années 1930 au milieu des années 1950, avant d'être démantelé par la répression anticommuniste de l'appareil d'état étatsunien, a multiplié les écrits, les discours et les actions pour sortir les femmes noires de la marginalité sociale et politique où elles étaient reléguées. Les militantes qui en sont issues ont fait de la condition des domestiques en particulier un problème emblématique des questions sociales et raciales, et les ont placées au centre du débat partout où elles étaient présentes au sein du mouvement ouvrier et du mouvement de libération noir. Au sein de la rédaction du journal Freedom notamment, Alice Childress a mis l'accent sur leur représentation, leur 
reconnaissance sociale et l'amélioration de leurs conditions de travail en mettant en scène le personnage de Mildred qui défend ses intérêts face à ses patronnes, prône la justice sociale et la paix et inspire d'autant plus celles et ceux qui lisent ses histoires qu'elle échappe aux stéréotypes de la militante. En 1966, lors d'une conférence à laquelle elle participe à propos de la représentation des femmes noires en littérature, la dramaturge continue à affirmer le lien indispensable entre engagement politique et création artistique sur ce sujet. Elle déclare que « la femme noire prendra sa place légitime dans la littérature américaine lorsque celles et ceux d'entre nous qui se soucient de la vérité, de la justice et d'une vie meilleure raconteront son histoire tout en connaissant et reconnaissant pleinement sa lutte constante et acharnée contre le racisme et pour les droits humains ${ }^{53} »$. Malgré des contributions majeures, comme celles de Toni Morrison - pour ne citer qu'elle - qui publie son premier roman, The Bluest Eye, quatre ans après la déclaration de Childress, la production culturelle destinée au grand public aux États-Unis compte jusqu'ici peu d'œuvres dans lesquelles la place des femmes noires est centrale. Et quand en 2009 un livre comme The Help écrit par Kathryn Stockett se penche sur la condition des domestiques noires vivant dans le Mississippi au début des années 1960, c'est pour apporter la preuve que ce sujet peut encore être traité sous la forme d'un roman d'apprentissage dont l'héroöne est une femme blanche sans qui ces dernières ne pourraient pas faire entendre leur voix. De ce fait, soixante-dix ans plus tard, le projet esthétique et politique à l'origine du personnage de Mildred reste marginal mais précurseur.

\section{BIBLIOGRAPHIE}

Alan, Robert, « Paul Robeson - the Lost Shepherd », The Crisis, novembre 1951.

Baker, Ella et Cooke, Marvel, « The Bronx Slave Market ! », The Crisis, novembre 1935.

Childress, Alice, « Why We Need a Union », Baltimore Afro-American, 14 juin 1958.

Childress, Alice, « Conversation From Life », Freedom, septembre 1951 - août 1955.

Childress, Alice, Like one of the Family: Conversations from a Domestic's life, New York, Independence Publishers, 1956.

Alice Childress Papers, « Writings », « Like One of the Family, Draft of Introduction by Trudier Harris (Annotated by AC) », Beacon Press, Reviews, 1986, série 649, n 45, dossier 45/2, Schomburg Center for Research in Black Culture.

Collier Brown, Jean, The Negro Woman Worker, Washington, Government Printing Office, 1938.

Cooke, Marvel, « Modern Slaves », Amsterdam News, 16 octobre 1937.

Cooke, Marvel, « I Was Part of the Bronx Slave Market », The Daily Compass, 8 Janvier 1950.

Cooke, Marvel, « Where Men Prowl and Women Prey on Needy Job-Seekers », The Daily Compass, 9 Janvier 1950. 
Cooke, Marvel, « Paper Bag Brigade Learns to Deal with Gypping Employers », The Daily Compass, 10 Janvier 1950.

Cooke, Marvel, « "Mrs. Legree” Hires on the Street, Always "Nice” Girls », The Daily Compass, 11 Janvier 1950.

Cooke, Marvel, « Some Ways to Kill the Slave Market », The Daily Compass, 12 Janvier 1950.

James E. Jackson and Esther Cooper Jackson Papers 1917-2004, « The Negro Woman Domestic Worker in Relation to Trade Unionism », M.A, Fisk University, 1940, série 347, n 8, dossier 31, Tamiment Library and Robert F. Wagner Labor Archives.

Currie, Kathleen, The Washington Press Club Foundation, Oral History Project Women in Journalism, Marvel Cooke interview, août 1990. http//www.wpcf.org/marvel-cooke/, page consultée le 21 février 2020.

Dale, Thelma, « Reconversion and the Negro People », Political Affairs, octobre 1945.

Freedom (1951-1955). Freedom Associates, Tamiment Library \& Robert F. Wagner Labor Archives, http://dlib.nyu.edu/freedom/, page consultée le 28 février 2020.

Hansberry, Lorraine, « Negroes Cast in Same Old Roles in TV Shows », Freedom, juin 1951.

Hansberry, Lorraine, « Women Voice Demands in Capital Sojourn », Freedom, octobre 1951.

Jones, Claudia, « An End to the Neglect of the Problems of the Negro Woman! », in Beverly GuySheftall, ed, Words of Fire: An Anthology of African-American Feminist Thought. New York : New Press, 1995, p. 108-123.

Jones, Claudia, « It Was a Sultry Sunday, But 50 Women Stuck It Out! », Sunday Worker, 10 août 1947.

Morrison, Toni, The Bluest Eye, New York, Holt Rinehart \& Winston, 1970.

Morrison, Toni, What Moves at the Margin: Selected Nonfiction, Jackson, University Press of Mississippi, 2008.

National Negro Congress Papers, « The Status of Negro Women », Thelma Dale, 3 février 1947, microfilm 6, partie II, bobine 34, Schomburg Center for Research in Black Culture.

Parti communiste des États-Unis, National Negro Congress 1937-1941, Résolutions du National Negro Congress, « Femmes », tenu à Chicago les 14, 15 et 16 février 1936, série 132, n 110 , dossier 19, Tamiment Library and Robert F. Wagner Labor Archives.

Pidgeon, Mary, « Employment of Women in the Early Postwar Period with Background of Prewar and War Data », Women's Bureau Bulletin, No. 211, 1946.

Richards, Beah E., A Black Woman Speaks and Other Poems, Los Angeles, Inner City Press, 1974.

Stockett, Kathryn, The Help, New York, Putnam, 2009.

Louise Thompson Patterson Papers, A Call to Negro Women, Sojourners for Truth and Justice, Sojourners' Organization, série 1, $\mathrm{n}^{\circ} 12$, dossier 17, Stuart A. Rose Manuscript, Archives, and Rare Book Library, Emory University.

Thompson, Louise, « Towards a Brighter Dawn », Woman Today, avril 1936.

Wright, Sarah. « The Negro Woman In American Literature », Freedomways, 6, n 1, hiver 1966 : 8-25.

Anderson, Karen Tucker, « Last Hired, First Fired: Black Women Workers during World War II », Journal of American History, 69, n 1, 1982, 82- 97. 
Boyce Davies, Carole, Left of Karl Marx: The Political Life of Black Communist Claudia Jones, Durham, Duke University Press, 2007.

Boyce Davies, Carole, Claudia Jones : Beyond Containment, Banbury, Ayebia Clarke Publishing, 2011. Curie, Fabien, « La NAACP et le Parti communiste face à la question des droits civiques, 1929-1941 », Thèse d'Études anglophones, Université de Strasbourg, 2013, https://tel.archivesouvertes.fr/tel-01059773/document, page consultée le 19 février 2020.

Clark-Lewis, Elisabeth, Living In, Living Out. African American Domestics and the Great Migration, New York, Kodansha International, 1996.

Denning, Michael, The Cultural Front: The Laboring of American Culture in the Twentieth Century, London, Verso, 2000.

Dolinar, Brian, The Black Cultural Front: Black Writers and Artists of the Depression Generation, Jackson, University Press of Mississippi, 2012.

Gellman, Erik S., Death Blow to Jim Crow: The National Negro Congress and the Rise of Militant Civil Rights, Chapel Hill, University of North Carolina Press, 2012.

Gore, Dayo F., Radicalism at the Crossroads: African American Women Activists in the Cold War, New York, New York University Press, 2011.

Harris, Trudier, From Mammies to Militants: Domestics in Black American Literature, Philadelphia, Temple University Press, 1982.

Horne, Gerald, Paul Robeson: The Artist as Revolutionary, London, Pluto Press, 2016.

Lamphere, Lawrence, « Paul Robeson, Freedom Newspaper, and the Black Press », PhD, Boston College, 2003.

Le Dantec-Lowry, Hélène, «Bonnes noires chez patrons blancs : conquérir un espace de liberté malgré tout ", 183-204, in Les femmes et l'espace, Résonances 7, Claude Cohen-Safir, dir., septembre 2003.

McDonald, Kathlene, Feminism, the Left, and Postwar Literary Culture, Jackson, University Press of Mississippi, 2012.

McDuffie, Erik, Sojourning for Freedom: Black Women, American Communism, and the Making of Black Left Feminism, Durham, N.C., Duke University Press, 2011.

Sharpless. Rebecca, Cooking in Other Women's Kitchens. Domestic Workers in the South, 1865-1960, Chapel Hill, The University of North Carolina Press, 2010.

Washington, Mary Helen, « Alice Childress, Lorraine Hansberry and Claudia Jones : Black Women Write the Popular Front ", in Bill V. Mullen et James Edward Smethurst eds, Left of the Color Line: Race, Radicalism, and Twentieth-Century Literature of the United States, Chapel Hill and London, The University of North Carolina Press, 2003.

Washington, Mary Helen, The Other Blacklist: the African American Literary and Cultural Left of the 1950s, New York, Columbia University Press, 2013.

\section{NOTES}

1. Toni Morrison, What Moves at The Margin: Selected Nonfiction, Jackson, University Press of Mississippi, 2008, p. 16. 
2. Voir Mary Helen Washington, « Alice Childress, Lorraine Hansberry and Claudia Jones: Black Women Write the Popular Front ", in Bill V. Mullen et James Edward Smethurst, eds, Lft of the Color Line: Race, Radicalism, and Twentieth-Century Literature of the United States, Chapel Hill and University of North Carolina Press, 2003, p. 183-204.

3. Voir Robert Alan, « Paul Robeson - the Lost Shepherd», The Crisis, novembre 1951, p. 569-73, et Lawrence Lamphere, " Paul Robeson, Freedom Newspaper, and the Black Press ", PhD, Boston College, 2003, p. 3 et 8.

4. Voir l'éditorial du premier numéro : «A New Voice », Freedom, novembre 1950, p. 4.

5. amphere, op. cit., p. 136.

6. Lamphere, op. cit., p. 6.

7. Pour une étude détaillée du mythe de la mammy, voir Hélène Le Dantec-Lowry, « Bonnes noires chez patrons blancs : conquérir un espace de liberté malgré tout ", 183-204, in Les femmes et l'espace, Résonances 7, Claude Cohen-Safir, dir., septembre 2003, p. 193-196.

8. Voir la rubrique d'avril 1952 intitulée "She's Like One of the Family» dans laquelle sa patronne Mrs. C. dit en présence de Mildred à son sujet à l'une de ses invitées : «C'est comme si elle faisait partie de la famille!».

9. Voir à ce sujet, en plus des ouvrages de Mary Helen Washington, Carole Boyce Davies, Left of Karl Marx: The Political Life of Black Communist Claudia Jones, Durham, Duke University Press, 2007, et Claudia Jones : Beyond Containment, Banbury, Ayebia Clarke Publishing, 2011 ; Erik McDuffie, Sojourning for Freedom : Black Women, American Communism, and the Making of Black Left Feminism, Durham, N.C., Duke University Press, 2011 ; Dayo F. Gore, Radicalism at the Crossroads : African American Women Activists in the Cold War, New York, New York University Press, 2011.

10. Toutes les citations en anglais ont été traduites par les soins de l'auteur de cet article.

11. Erik S. Gellman, Death Blow to Jim Crow: The National Negro Congress and the Rise of Militant Civil Rights, Chapel Hill, University of North Carolina Press, 2012.

12. Voir Fabien Curie, La NAACP et le Parti communiste face à la question des droits civiques, 1929-1941, Thèse d'Études anglophones, Université de Strasbourg, 2013, p. 183-203 et 242-275, https://tel.archives-ouvertes.fr/tel-01059773/document, page consultée le 19 février 2020.

13. Jean Collier BROWN, The Negro Woman Worker, Washington, Government Printing Office, 1938, p. 2.

14. Voir Rebecca Sharpless, Cooking in Other Women's Kitchens. Domestic Workers in the South, 1865-1960, Chapel Hill, The University of North Carolina Press, 2010, p. 86. L'auteure y présente l'effroi de nombreuses domestiques quand elles apprennent qu'elles restent exclues des réformes du Social Security Act et cite la lettre de protestation d'une cuisinière de Washington D.C., Anna Smith, adressée directement à la première dame Eleanor Roosevelt.

15. Résolutions du National Negro Congress, «Femmes ", tenu à Chicago les 14, 15 et 16 février 1936, National Negro Congress 1937-1941, série 132, n 110, dossier 19, p. 5-16, Parti communiste des États-Unis, Tamiment Library and Robert F. Wagner Labor Archives.

16. Née à Chicago, Louise Thompson (1901-1999) s'installe à New York à la fin des années 1920, où elle fréquente de nombreuses personnalités de la Renaissance de Harlem. Elle rejoint le CPUSA au début des années 1930, au moment où elle joue un rôle important dans l'animation de la campagne de mobilisation pour la libération des neuf de Scottsboro. Responsable de l'International Workers Order (IWO), une société d'assurance mutuelle, elle participe à la création du National Negro Congress (NNC). Mariée en 1940 à William Patterson, un dirigeant noir du CPUSA, elle reste une militante fidèle au parti jusqu'à sa mort.

17. Louise Thompson, « Towards a Brighter Dawn », Woman Today, avril 1936, p. 14.

18. Iid.

19. Voir Ella Baker et Marvel Cooke, «The Bronx Slave Market! », The Crisis, novembre 1935. Ella Baker (1903-1986) était une militante de la Southern Christian Leadership Conference aux côtés 
de Martin Luther King et a également été à l'origine de la création du Student Nonviolent Coordinating Committee.

20. Voir ses cinq articles parus dans le Daily Compass du 8 au 12 janvier 1950.

21. Vir Kathleen Currie, The Washington Press Club Foundation, Oral History Project Women in Journalism, Marvel Cooke interview, août 1990, p. 76, 83 et 123. Fondé à New York en 1934, le DWU est animé par la syndicaliste africaine-américaine Dora Lee Jones. En 1937, il compte 350 membres, dont $75 \%$ sont noires, qu'il mobilise pour faire pression sur l'assemblée de l'état de New York et imposer un contrat type de soixante heures de travail sur six jours par semaine et un salaire minimum de quinze dollars. Voir Marvel Cooke, « Modern Slaves », Amsterdam News, 16 octobre 1937.

22. "The Negro Woman Domestic Worker in Relation to Trade Unionism », M.A., Fisk University, 1940, série 347, n 8, dossier 31, p. 83, James E. Jackson and Esther Cooper Jackson Papers 1917-2004, Tamiment Library and Robert F. Wagner Labor Archives.

23. id, p. 40 et 98 .

24. id, p. 14-15.

25. Voir « Say slave market conditions exist », Afro-American, 21 décembre 1940, p. 16.

26. "The Status of Negro Women », Thelma Dale, 3 février 1947, microfilm 6, partie II, bobine 34, p. 2, National Negro Congress Papers, Schomburg Center for Research in Black Culture. Voir également à ce sujet Thelma Dale, «Reconversion and the Negro People », Political Affairs, octobre 1945, p. 894.

27. Caudia Jones, « An End to the Neglect of the Problems of the Negro Woman! in Beverly GuySheftall, ed, Words of Fire: An Anthology of African-American Feminist Thought, New York, New Press, 1995, p. 116 et 111.

28. Iid, p. 120.

29. Voir Dayo F. Gore, Radicalism at the Crossroads: Arican American Women Activists in the Cold War, New York et London, New York University Press, 2011, p. 66-68.

30. Voir Brian Dolinar, The Black Cultural Front: Black Writers and Artists of the Depression Generation, Jackson, University Press of Mississippi, 2012, p. 64 et 66.

31. Voir Gerald Horne, Paul Robeson: The Artist as Revolutionary, London, Pluto Press, 2016, p. 134.

32. Voir entre autres Lorraine Hansberry, «Women Voice Demands in Capital Sojourn », Freedom, Octobre 1951, p. 6.

33. Voir « A Call to Negro Women, Sojourners for Truth and Justice », Sojourners' Organisation, série $1, \mathrm{n}^{\circ} 12$, dossier 17, Louise Thompson Patterson Papers, Stuart A. Rose Manuscript, Archives, and Rare Book Library, Emory University.

34. Voir " A New Voice", op. cit.: «FREEDOM se fera le porte- voix des pauvres et des déshérité.e.s parmi nous ».

35. Mary Helen Washington, The Other Blacklist: The African American Literary and Cultural Left of the 1950s, New York, Columbia University Press, 2013, p. 142.

36. Mary Helen Washington, "Alice Childress, Lorraine Hansberry and Claudia Jones: Black Women Write the Popular Front », op.cit., p. 164.

37. Voir « U.S. Arts Contest on Peace Theme Set Deadline June 1 », Sunday Worker, 13 mai 1951, p. 7.

38. Beah E. Richards, A Black Woman Speaks and Other Poems, Los Angeles, Inner City Press, 1974.

39. Voir Kathlene McDonald, Feminism, the Left, and Postwar Literary Culture, Jackson, University Press of Mississippi, 2012, p. 66.

40. Michael Denning, The Cultural Front: The Laboring of American Culture in The Twentieth Century, London, Verso, 2000, p. 228.

41. Ibid, p. 229.

42. Ibid, p. 65.

43. «It Was a Sultry Sunday, But 50 Women Stuck It Out! », Sunday Worker, 10 août 1947, p. 9. 
44. Lorraine Hansberry, "Negroes Cast in Same Old Roles in TV Shows ", Freedom, juin 1951, p. 7. 45. Voir "Conversation From Life", Freedom, novembre 1952, où Mildred offre à ses voisin.e.s pour Noël la nourriture qu'ils et elles n'ont pas les moyens de se payer le reste de l'année.

46. Voir « Conversation From Life », Freedom, avril 1952, p. 8, et aussi « Douglass Center Prepares Program », New York Amsterdam News, 19 avril 1952, p. 20, qui annonce la participation d'Alice Childress à une conférence sur la façon dont le peuple noir a utilisé l'humour comme une arme dans les luttes pour sa libération.

47. Mary Helen Washington, The Other Blacklist: The African American Literary and Cultural Left of the 1950s, New York, Columbia University Press, 2013, p. 146.

48. Voir « Conversation From Life », Freedom, octobre 1952, p. 2 et 8.

49. Voir Nena Evans, «Leader of Domestic Workers », Freedom, septembre 1952, p. 2.

50. Il s'agit d'une annotation de Childress sur les épreuves de l'introduction de la seconde édition de son livre écrite par Trudier Harris. Voir "Writings ", "Like One of the Family, Draft of Introduction by Trudier Harris (Annotated by AC) », Beacon Press, Reviews, 1986, série 649, n 45, dossier 45/2, p. 1, Alice Childress Papers, Schomburg Center for Research in Black Culture.

51. Ibid, p. 8.

52. Ibid, p. 18. Voir aussi Elisabeth Clark-Lewis, Living In, Living Out. African American Domestics and the Great Migration, New York, Kodansha International, 1996 et Rebecca Sharpless, Cooking in Other Women's Kitchens. Domestic Workers in the South, 1865-1960, Chapel Hill, The University of North Carolina Press, 2010 : les deux ouvrages fournissent de nombreux exemples de ruses, manœuvres et autres actes de protestation de la part de domestiques avec lesquelles Lewis et Sharpless se sont entretenues.

53. The Negro Woman in American Literature, Freedomways, hiver 1966, p. 19.

\section{RÉSUMÉS}

Cet article examine la place des domestiques noires aux États-Unis entre le milieu des années 1930 et le milieu des années 1950 ainsi que les questions liées à la lutte pour l'amélioration de leurs conditions de travail et pour leur reconnaissance sociale, une lutte qui a lieu sur les lieux de travail via le développement du syndicalisme mais aussi dans la sphère socioculturelle où les logiques de ségrégation raciale et de suprématie blanche prédominent. En particulier, les femmes noires membres ou sympathisantes du Parti communiste américain constituent un courant militant désigné sous le nom de Black Left Feminism (féminisme noir de gauche) qui permet de sortir les domestiques de l'ombre et du silence et de porter un sujet jusqu'ici marginal au centre des débats des organisations du mouvement ouvrier et en faveur des droits civiques auxquelles elles appartiennent. Dans ce cadre, la contribution politique et artistique de la dramaturge Alice Childress mérite une attention toute particulière. Conversation From Life, sa série fictionnelle rédigée chaque mois de 1951 à 1955 pour le journal de la gauche noire Freedom, est la première tentative de représentation d'une domestique qui défend en même temps fierté noire, dignité ouvrière et pouvoir d'agir féminin dans un contexte de guerre froide où toute démarche de contestation est férocement réprimée. Mildred, le personnage principal, est le symbole du passage possible des femmes noires de la marge à l'avant-garde des luttes qui caractérisent cette période, en faveur des droits des noir.e.s, des salarié.e.s, des femmes et de la paix. 
This paper examines the position of black women domestic workers in the United States from the mid-1930s to the mid-1950s and their struggles for improved working conditions and social recognition, which primarily took place in the workplace through the development of trade unionism, as well as in the sociocultural sphere where the logics of racial segregation and white supremacy were predominant. Specifically, black women who were members or sympathizers of the Communist Party of the United States of America (CPUSA) constituted a militant movement known as Black Left Feminism that placed black domestics at the heart of their analysis and artistic expression. This movement brought what was a hitherto marginal subject out of the shadows into the center of debate within the labor and civil rights organizations to which they belonged. In this context, playwright Alice Childress's political and artistic contributions deserve special attention. From 1951 to 1955, Childress wrote a monthly fiction column titled Conversation From Life for the black left newspaper Freedom. Conversation From Life was the first attempt to depict a domestic worker who simultaneously defends black pride, workers' dignity and women's power to act, in spite of the repressive Cold War context that dominated U.S. society at the time. Mildred, the main character, symbolizes the possible transition of black women from the margins to the vanguard of the struggles for black people's, workers', and women's rights, and for peace.

Este artículo examina el lugar que ocupaban las trabajadoras domésticas negras en los Estados Unidos entre mediados de la década de 1930 y mediados de la década de 1950 y las cuestiones relacionadas con la lucha por mejorar sus condiciones de trabajo y su reconocimiento social. Una lucha que tuvo lugar en el entorno laboral a través del desarrollo del sindicalismo, pero también en la esfera cultural donde predominaban las lógicas de la segregación racial y la supremacía blanca. La participación de las mujeres negras miembros o simpatizantes del Partido Comunista Americano y su constitución como corriente militante conocida como Black Left Feminism permite sacar a las empleadas domésticas de las sombras y del silencio y poner en el centro de los debates de las organizaciones del movimiento obrero y a favor de los derechos civiles a los que pertenecían un tema hasta ahora marginal. En este contexto, la contribución política y artística de la dramaturga Alice Childress merece una atención especial. Su columna Conversation From Life, escrita cada mes para el periódico Freedom de 1951 a 1955, es el primer intento de describir a una trabajadora doméstica que defiende simultáneamente el orgullo negro, la dignidad de los trabajadores y el empoderamiento de las mujeres para actuar en un contexto de Guerra Fría en el que todas las formas de protesta eran ferozmente reprimidas. Mildred, la protagonista, es el símbolo de la posibilidad para las mujeres negras de dejar de ser marginadas para pasar a ser partícipe de la vanguardia de las luchas por los derechos de los negros, de los trabajadores y de las mujeres y por la paz que caracterizaban este período.

\section{INDEX}

Palabras claves : trabajadora doméstica, mujer negra, sindicalismo, McCarthyismo, Estados Unidos

Keywords : domestic worker, black woman, trade unionism, McCarthyism, United-States of America

Mots-clés : Domestique, femme noire, syndicalisme, maccarthysme, États-Unis 


\section{AUTEUR}

\section{GRÉGORY BEKHTARI}

Grégory Bekhtari est doctorant en histoire et culture américaines à l'Université de Paris Nanterre sous la direction de Caroline Rolland-Diamond. Il travaille à une thèse qui ambitionne de retracer la généalogie intellectuelle et politique du Black Feminism, courant militant et théorique qui naît à la fin des années 1960 aux États-Unis, où il se développe jusqu'au milieu des années 1980. Il est rattaché au Centre de recherches anglophones (CREA), Université Paris Nanterre, 92001, Nanterre Cedex, France. (gbekhtari@parisnanterre.fr) 